\title{
Cement-Based Materials Containing Graphene Oxide and Polyvinyl Alcohol Fiber: Mechanical Properties, Durability, and Microstructure
}

\author{
Wenguang Jiang ${ }^{1}$, Xiangguo Li ${ }^{1, *}$, Yang $\mathrm{Lv}^{1}{ }^{1}$, Mingkai Zhou ${ }^{1}$, Zhuolin Liu ${ }^{1}$, Zhaofeng Ren ${ }^{1}$ \\ and Zhuqing $\mathrm{Yu}^{2}$ \\ 1 State Key Laboratory of Silicate Materials for Architectures, Wuhan University of Technology, \\ Wuhan 430070, China; JWG930329@163.com (W.J.); yang.lv@whut.edu.cn (Y.L.); \\ zhoumingkai@163.com (M.Z.); lin7336@126.com (Z.L.); RZF15671660187@163.com (Z.R.) \\ 2 College of Materials Science and Engineering, Nanjing Tech University, Nanjing 210000, China; \\ zyu@njtech.edu.cn \\ * Correspondence: lixiangguo@whut.edu.cn; Tel.: +86-27-87214969
}

Received: 18 July 2018; Accepted: 16 August 2018; Published: 21 August 2018

\begin{abstract}
The influence of graphene oxide (GO) and polyvinyl alcohol (PVA) fiber on the mechanical performance, durability, and microstructure of cement-based materials was investigated in this study. The results revealed that compared with a control sample, the mechanical strength and durability of cement-based materials were significantly improved by adding PVA fiber and GO. The compressive and flexural strength at $28 \mathrm{~d}$ were increased by $30.2 \%$ and $39.3 \%$, respectively. The chloride migration coefficient at $28 \mathrm{~d}$ was reduced from $7.3 \times 10^{-12} \mathrm{~m}^{2} / \mathrm{s}$ to $4.3 \times 10^{-12} \mathrm{~m}^{2} / \mathrm{s}$. Under a sulfate corrosion condition for $135 \mathrm{~d}$, the compressive and flexural strength still showed a $13.9 \%$ and $12.3 \%$ gain, respectively. Furthermore, from the Mercury Intrusion Porosimetry (MIP) test, with the incorporation of GO, the cumulative porosity decreased from more than $0.13 \mathrm{~cm}^{3} / \mathrm{g}$ to about $0.03 \mathrm{~cm}^{3} / \mathrm{g}$, and the proportion of large capillary pores reduced from around $80 \%$ to $30 \%$ and that of medium capillary pores increased from approximately $20 \%$ to $50 \%$. Scanning electron microscope (SEM) images showed a significant amount of hydration products adhering to the surface of PVA fiber in the GO and PVA fiber modified sample. The addition of GO coupling with PVA fiber in cement-based materials could promote hydration of cement, refine the microstructure, and significantly improve mechanical strength and durability.
\end{abstract}

Keywords: graphene oxide; PVA fiber; cement-based materials; mechanical strength; durability; microstructure

\section{Introduction}

As the main ingredients of concrete and mortar, cement has been widely used in buildings and structures [1]. However, both the concrete and mortar are poor in the tensile strength, strain capacity, and durability due to their brittle cracking defects and loose structure [2]. With the progress of society, traditional cement-based materials are unable to meet the increasing demands for durability and mechanical properties of buildings and structures. Therefore, it is significant for the use of cement-based materials if these issues can be effectually solved. Recently, high-performance and high-durability cement-based materials have been developed rapidly through the use of modification materials such as fibers and nanomaterials to improve the structural functionality [3,4].

Fibers such as steel fiber, basalt fiber (BF), polypropylene (PP) fiber, and polyvinyl alcohol (PVA) fiber are common modification materials used in cement-based materials. It has been proven that the toughness and crack resistance of cement-based materials can be effectively improved by incorporating 
fibers [5-11]. The toughening and reinforcing effect of fibers in cement-based materials is closely related to the bonding properties between the fibers and the cementitious components [5,8-11]. PVA fiber has been proved to be a good toughening and reinforcing admixture for cement-based materials due to its excellent performance in improving mechanical strength [12]. This is because, on the one hand, PVA fiber possesses a high tensile strength, and on the other hand, its hydrophilic surface interacts with cementitious component and could form a strong bonding combination [13-16]. However, PVA fiber has not yet been demonstrated to prevent microcracks formation or refine pore structure. It was incapable for significantly improving the durability of cement-based materials.

With the rise of nanotechnology, the application of nanomaterials in cement-based materials for improving properties has become a new research hotspot in recent years [17]. Nanomaterials can fill the voids in cement-based materials and form tighter interfaces between aggregates and cementitious components, which is beneficial to the improvement of mechanical properties and durability [18-20]. Nano silica (NS) with a large surface area can increase the pozzolanic activity by acting as a center for crystallization, resulting in the formation of large idiomorphic crystals of Ca-Si composition, thus leading to denser microstructure with reduced porosity and increased mechanical strength [21-23]. Carbon nanotubes (CNTs) in cement-based materials could lower the porosity and improve compressive and tensile strength by more than 30\% [24]. Unlike other nanomaterials, graphene oxide (GO), the graphene derivative, has excellent mechanical properties whose Young's modulus and intrinsic strength are evaluated as high as $1 \mathrm{TPa}$ and $100 \mathrm{GPa}$, respectively [25]. Moreover, owing to the existence of abundant hydrophilic oxygen-containing functional groups, such as hydroxyl, carbonyl, and carboxyl, GO could be dispersed evenly in water [26]. There are several articles that have reported the modification effects of GO on cement-based materials. The research [27] of our group showed that, based on the isothermal calorimeter test, GO could accelerate the hydration rate of cement. Li et al. [28] also reported that the hydration rate of cement was observed to increase with the increase of GO content. Pan et al. [29] showed that with the incorporation of GO, the compressive strength and flexural strength of cement paste increased by $15-33 \%$ and $41-59 \%$, respectively. Lv et al. [30] revealed that with the addition of $0.03 \%$ GO, the tensile strength, flexural strength, and compressive strength of cement composites increased by $78.6 \%, 60.7 \%$, and $38.9 \%$, respectively. This can be explained by the function groups of GO providing attachment sites for water and cement, thus acting as the crystal nucleuses for cement hydrates and promoting the hydration rate [31]. The durability of cement-based materials could also be improved by GO introduction. Mohammed et al. [32] demonstrated that the pore structure of cement-based materials could be refined by GO, as a result, the durability increased significantly, and the experimental results also revealed that the cement matrix exhibited high freeze-thaw resistance in the case of GO content.

In summary, PVA fiber has a unique effect on the toughness of cement based materials while GO is beneficial to the improvement of the mechanical strength and durability. Thus, a substantial improvement in comprehensive performance of cement-based materials is expected by utilizing the synergy effect of PVA fiber and GO. The purpose of this study is to investigate the mechanical performances, chloride migration, sulfate corrosion resistance, and drying shrinkage property of cement-based materials modified by GO or/and PVA fiber. Moreover, the synergy effect of GO and PVA fiber on the modified cement-based materials was discussed by means of a pore structure test and scanning electron microscope (SEM) images analysis.

\section{Experiment}

\subsection{Materials}

GO was prepared in the laboratory, the preparation process and characteristics were described in the previous work [33]. The Fourier-transform infrared spectroscopic (FT-IR) spectrum (Thermo Nicolet, Madison, WI, USA) confirmed that the oxygen functional groups of $-\mathrm{OH},-\mathrm{COOH}$, and -Owere found on the surface of GO. The X-ray diffraction (XRD) spectra (RIGAKU Corporation, Tokyo, 
Japan) showed the diffraction peak of GO was at $2 \theta=9.71^{\circ}$, and the corresponding interlayer spacing was $0.93 \mathrm{~nm}$. In addition, an atomic force microscope (AFM) image (Asylum Research, Santa Barbara, CA, USA) of GO is displayed in Figure 1. It can be seen from the image that the size of the GO sheet reached the nanometer scale, and the thickness of a single irregular sheet was about $1 \mathrm{~nm}$, with its length and width at about $1 \mu \mathrm{m}$ and $2.5 \mu \mathrm{m}$, respectively.

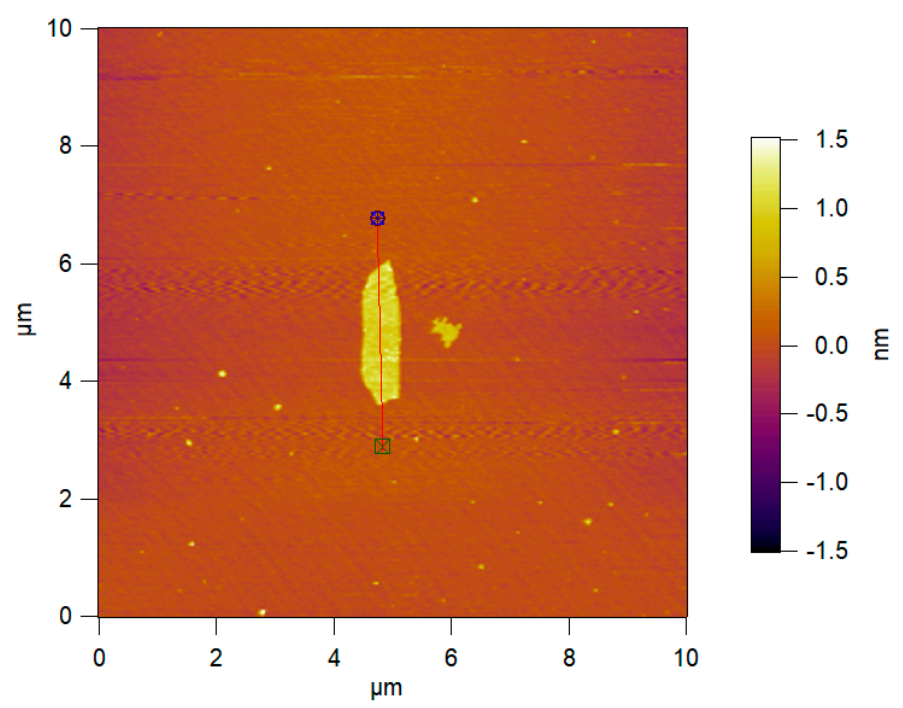

(a)

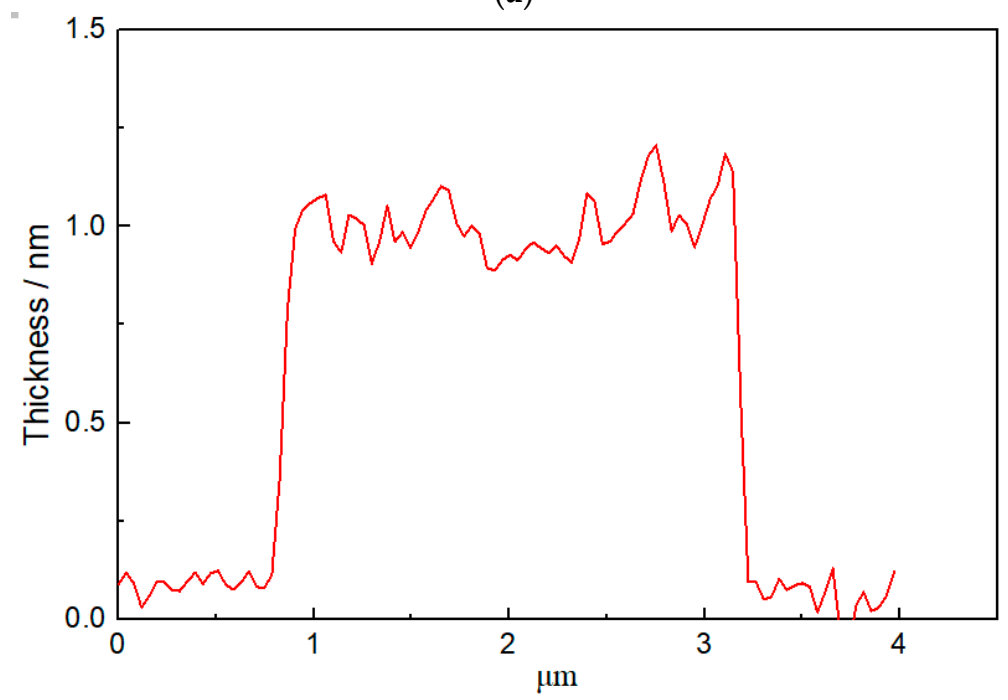

(b)

Figure 1. Atomic force microscope (AFM) image of the graphene oxide (GO) (a) The length and width of GO; (b) The thickness of GO.

Ordinary Portland Cement (OPC) CEM I 42.5, fly ash (FA), and silica fume (SF) used in the present study were supplied by China Resources Cement Holdings Limited (Wuhan, China), Huaneng Yangluo Power Plant (Wuhan, China) and Wuhan Sentai metallurgy Co. Ltd. (Wuhan, China), respectively. Table 1 presented the chemical compositions of the materials obtained by X-Ray Fluorescence (XRF) (PANalytical B.V., Almelo, The Netherlands) test.

The siliceous sand used in this study was supplied by Xiamen China ISO Standard Sand Co. Ltd. (Xiamen, China) PVA fiber with $12 \mathrm{~mm}$ length was obtained from Wuhan Tianhui fiber material Co. Ltd. (Wuhan, China) Polycarboxylate (PC) superplasticizer with $40 \%$ solid content and defoaming agents 
(DA, tributyl phosphate) were produced by Wuhan Huaxuan High-Tech Co. Ltd. (Wuhan, China). The deionized water was used for all mixtures.

Table 1. Chemical composition of ordinary Portland cement (OPC), fly ash (FA), and silica fume (SF).

\begin{tabular}{cccc}
\hline Compositions & OPC & FA & SF \\
\hline $\mathrm{SiO}_{2}$ & 21.99 & 51.53 & 89.63 \\
$\mathrm{Al}_{2} \mathrm{O}_{3}$ & 5.92 & 27.78 & 0.12 \\
$\mathrm{Fe}_{2} \mathrm{O}_{3}$ & 3.26 & 4.73 & 0.35 \\
$\mathrm{CaO}$ & 58.64 & 5.21 & 3.21 \\
$\mathrm{MgO}$ & 1.98 & 1.03 & 2.82 \\
$\mathrm{~K}_{2} \mathrm{O}$ & 0.74 & 1.13 & 1.01 \\
$\mathrm{Na}_{2} \mathrm{O}$ & 0.27 & 0.62 & 0.42 \\
$\mathrm{SO}_{3}$ & 2.6 & 1.87 & 0.92 \\
Loss on ignition (LOI) & 3.5 & 3.16 & 1.16 \\
\hline
\end{tabular}

\subsection{Mixing Procedure and Sample Preparation}

Table 2 showed the mixture design for the mortar samples preparation. The amount of the mixing water was the sum of the deionized water and the water introduced by the PC superplasticizer. The dosage of PC referred to the amount of solids in the PC superplasticizer solution. For example, $6.2 \mathrm{~g}$ PC was equivalent to $15.5 \mathrm{~g}$ of PC superplasticizer solution and $260 \mathrm{~g}$ of mixing water that amounted to $250.7 \mathrm{~g}$ of deionized water plus $9.3 \mathrm{~g}$ of water introduced by the PC superplasticizer solution. In order to ensure uniform dispersion of GO, ultrasonic preprocessing was necessary. The specific process was as follows: First, the PC superplasticizer solution and DA were diluted into $75 \mathrm{wt} \%$ deionized water. Then, the weighed GO was added into the mixture solution and gently stirred with a glass rod. After that, the mixture solution was oscillated for $20 \mathrm{~min}$ using an ultrasonic machine (SB-5200D) (Ningbo Scientz Biotechnology Co., Ltd., Ningbo, China) with $250 \mathrm{~W}$ power and $40 \mathrm{KHz}$ frequency. Besides, the PVA fiber was mixed with sand and binder in a mortar mixer (JJ-5) (Wuxi Jianyi Experiment Instrument Co. Ltd., Wuxi, China) for $2 \mathrm{~min}$ at low speed (the rotation rate: $140 \pm 5 \mathrm{r} / \mathrm{min}$; the revolution rate: $62 \pm 5 \mathrm{r} / \mathrm{min}$ ). Then, the sonicated GO dispersion was added and the remnant was washed in by the remaining $25 \mathrm{wt} \%$ deionized water. Afterwards, the mixture was continually stirred for $1 \mathrm{~min}$ at low speed and $2 \mathrm{~min}$ at high speed (the rotation rate: $285 \pm 10 \mathrm{r} / \mathrm{min}$; the revolution rate: $125 \pm 10 \mathrm{r} / \mathrm{min}$ ). The experiment was carried out at $20{ }^{\circ} \mathrm{C}$.

Table 2. Mix proportion of cement-based materials. C0 represents the control sample, CF and CG represent the polyvinyl alcohol (PVA) fiber and GO modified sample, respectively. CFG represents the GO coupling with the PVA fiber modified sample. PC = polycarboxylate; DA = defoaming agents.

\begin{tabular}{cccccccccc}
\hline Samples & OPC/g & FA/g & SF/g & Sand/g & Water/g & GO/g & PVA/g & PC/g & DA/g \\
\hline C0 & 620 & 300 & 80 & 1000 & 260 & 0 & 0 & 6.2 & 0.5 \\
CF & 620 & 300 & 80 & 1000 & 260 & 0 & 5 & 6.2 & 0.5 \\
CG & 620 & 300 & 80 & 1000 & 260 & 0.5 & 0 & 6.2 & 0.5 \\
CFG & 620 & 300 & 80 & 1000 & 260 & 0.5 & 5 & 6.2 & 0.5 \\
\hline
\end{tabular}

\subsection{Characterization of the Cement-Based Materials}

\subsubsection{Mechanical Performance}

The mixtures were casted into $40 \times 40 \times 160 \mathrm{~mm}^{3}$ steel molds twice and vibrated on a vibrostand 60 times for densification after each casting. Then, the surface of the castings were smoothed with a scraper and covered with preservative film. Afterwards, they were placed for $24 \mathrm{~h}$ at a temperature of $20 \pm 5{ }^{\circ} \mathrm{C}$. After demolding, the specimens were cured under the standard curing condition at constant temperature and relative humidity $(\mathrm{RH})\left(20 \pm 2{ }^{\circ} \mathrm{C}\right.$ and $\left.\geq 95 \% \mathrm{RH}\right)$ until test age. The mechanical 
strength test was performed referring to Chinese standard GB/T 50081-2002 at the age of 3, 7, and $28 \mathrm{~d}$ [34]. Three samples were used for the flexural strength measurement and six samples left from the flexural test were used for the compressive strength measurement. The loading rates for flexural and compressive strength measurement were $50 \pm 10 \mathrm{~N} / \mathrm{s}$ and $2400 \pm 200 \mathrm{~N} / \mathrm{s}$, respectively. The mean value of three test results and six test results was obtained for the flexural and compressive strength of each mixture, respectively.

\subsubsection{Rapid Chloride Migration Test (RCM)}

The mixtures were casted into $\Phi 100 \times 50 \mathrm{~mm}^{2}$ plastic molds. The molding process and curing conditions were the same as that of the specimens for mechanical strength measurement. Curing for $28 \mathrm{~d}$, three samples for each mixture were used for the RCM test which was conducted in accordance with Chinese standard GB/T 50082-2009 [35]. In the preparatory phase, the specimens were placed in the vacuum container and the pressure within $1 \sim 5 \mathrm{KPa}$ was maintained for $3 \mathrm{~h}$. Saturated calcium hydroxide solution was added to immerse the specimens, and the vacuum level was maintained for one additional hour. Specimens were soaked in the added solution for $18 \pm 2 \mathrm{~h}$ after turning off the pump. In the test setup, the lateral surface of each specimen was sealed with a rubber sleeve and fixed by two stainless steel hoops (height $25 \mathrm{~mm}$ ). Then, $0.3 \mathrm{~mol} / \mathrm{L} \mathrm{NaOH}$ solution and $10 \% \mathrm{NaCl}$ solution were poured into the anode chamber and cathode chamber, respectively. The voltage was $30 \pm 0.2 \mathrm{~V}$ to record the initial current. Afterwards, the test voltage was adjusted and the test duration was set according to the initial current. Record the initial and final temperatures of anode solution and the final current. After the migration test, each sample was split into two halves and $0.1 \mathrm{~mol} / \mathrm{L} \mathrm{AgNO}_{3}$ solution was sprayed on the fracture surface to confirm the penetration depth of chloride ion.

\subsubsection{Sulfate Corrosion Resistant}

After standard curing for $28 \mathrm{~d}$, the mortar specimens with a size of $40 \times 40 \times 160 \mathrm{~mm}^{3}$ were immersed in a $5 \% \mathrm{Na}_{2} \mathrm{SO}_{4}$ solution. After immersion for 45, 90, and $135 \mathrm{~d}$, compressive and flexural strength measurements of three samples for each mixture were in accordance with the testing procedures in Section 2.3.1.

\subsubsection{Drying Shrinkage}

The mixtures were casted into $25 \times 25 \times 280 \mathrm{~mm}^{3}$ steel molds (both ends were embedded with gage stud bold) twice, scraped with a scraper, and mashed with a square tamper $\left(23 \times 23 \mathrm{~mm}^{2}\right)$ 24 times for densification after each casting. After curing in the mold (covered with preservative film) for $24 \mathrm{~h}$ at a temperature of $20 \pm 5{ }^{\circ} \mathrm{C}$, the specimens were demolded and stored in the room with constant temperature and relative humidity of $20 \pm 3{ }^{\circ} \mathrm{C}$ and $50 \pm 4 \%$. The length of the specimens was recorded immediately after demolding by a comparator (precision: $0.01 \mathrm{~mm}$ ) and was regarded as the initial length for the drying shrinkage calculation. The drying shrinkage of the specimens was measured up to 90 days after demolding. Three specimens were used for each mixture.

\subsubsection{Mercury Intrusion Porosimetry (MIP) analysis}

The pore structure characteristics of the cement-based materials at $28 \mathrm{~d}$ age were studied by MIP tests which were carried out on a Quanta Chrome Pore Master GT60 mercury intrusion porosimeter (Quanta Chrome, Boynton Beach, FL, USA). For the measurement, the specimens were broken into lumps with diameters ranging from 3 to $6 \mathrm{~mm}$. Afterwards, the lumps were soaked in anhydrous ethanol for more than $24 \mathrm{~h}$ to replace the pore water, and then dried at $40{ }^{\circ} \mathrm{C}$ for more than $12 \mathrm{~h}$ in a vacuum drying oven. During MIP, the high pressure was within the range of $140-420 \mathrm{KPa}$ and the low pressure was within the range of $1.5-350 \mathrm{KPa}$. Pore size ranging from $0.0035 \mu \mathrm{m}$ to $200 \mu \mathrm{m}$ can be recorded. 


\subsubsection{Microstructure Characterization}

Microstructure characteristics of the fracture surfaces of the cement-based materials were studied by scanning electron microscope (SEM) using a high resolution scanning electron microscopy (QUANTA 200 FEG, Field Electron and Ion Company (FEI), Hillsboro, OR, USA) equipped with a cold field emission electron gun operating at $15 \mathrm{kV}$. For the measurement, the specimens which were kept in anhydrous ethanol after being crushed at $28 \mathrm{~d}$ were dried at $40{ }^{\circ} \mathrm{C}$ for more than $12 \mathrm{~h}$ in a vacuum drying oven. Then, they were processed into lumps with a size of approximately $5 \times 5 \times 2 \mathrm{~mm}^{3}$. The fracture surface was sputter-coated with a thin layer $(1 \mathrm{~nm})$ of Pt prior to SEM observation.

\section{Results and Discussion}

\subsection{Mechanical Performances}

The compressive and flexural strength of cement-based materials modified by GO and/or PVA fiber are summarized in Figures 2 and 3, respectively. C0 represented the control sample, CF and CG represented the PVA fiber and GO modified sample, respectively. CFG represented the GO coupling with PVA fiber modified sample.

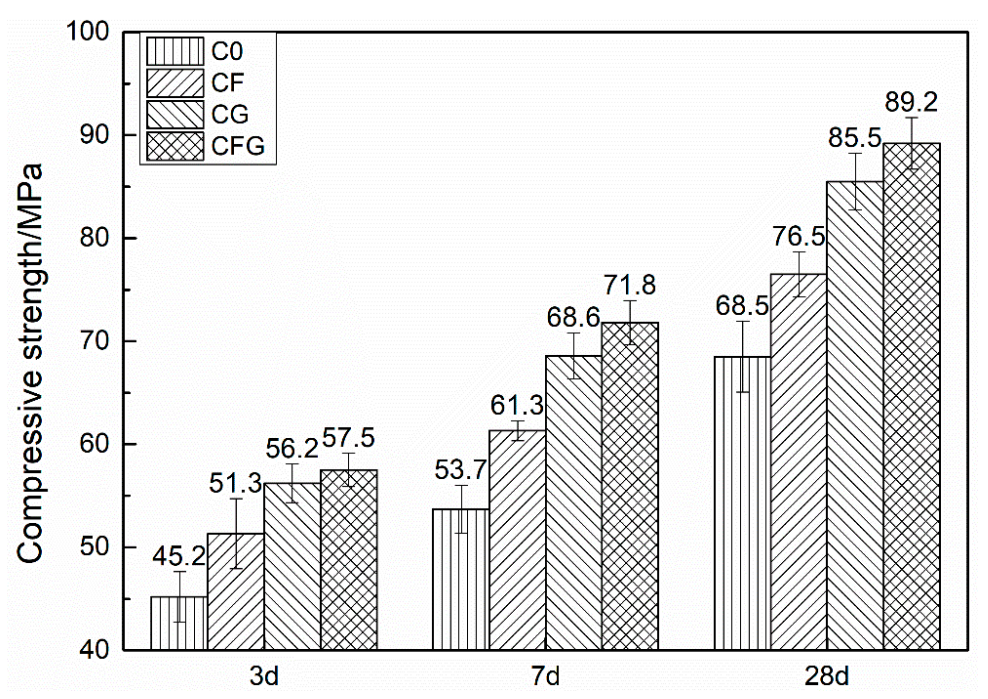

Figure 2. Compressive strength vs. curing time.

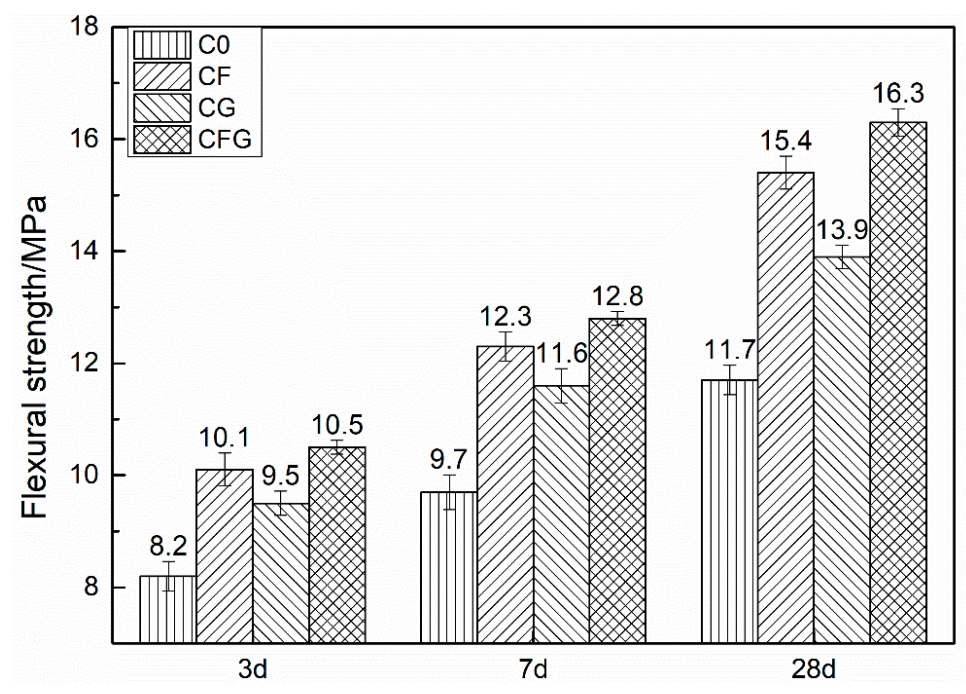

Figure 3. Flexural strength vs. curing time. 
It is obvious from the figures that the addition of PVA fiber and/or GO could increase both the compressive and flexural strength of the mixtures. The incorporation of PVA fiber into the mixtures lead to an increased compressive strength with an improvement of more than $10 \%$ at each age, and the flexural strength was increased by $23.2 \%$ and $31.6 \%$ at $3 \mathrm{~d}$ and $28 \mathrm{~d}$, respectively. It can be concluded that PVA fiber has a substantially more pronounced effect on the flexural strength than the effect on the compressive strength. According to the previous studies [36,37], PVA fiber can act as a bridge in cement-based materials, which not only inhibits the expansion of the crack but also consumes fracture energy in the process of being pulled out or tensile failure, leading to mechanical strength improvement, particularly the flexural strength. Differently, with the addition of GO into cement-based materials, the increment in compressive strength was more prominent. At GO content, the value of the increase rate for the compressive strength improvement was approximately $25 \%$ whereas for flexural strength improvement it was less than $20 \%$ at each age. As previously stated by Wang et al. [38], GO was involved in the cement hydration, accelerating the nucleation, growth, and phase separation process of hydration products, which promotes the hydration rate and improves the crystal order, thus enhancing the mechanical strength of the cement-based materials. When the cement-based materials were modified by PVA fiber coupling with GO, the compressive strength was increased by around $30 \%$ at each age. The value of the increase rate for the flexural strength improvement was from $28 \%$ to $39.3 \%$ with the curing age increasing from $3 \mathrm{~d}$ to $28 \mathrm{~d}$. The compressive strength was mainly increased by GO, whereas the flexural strength was increased both by GO and PVA fiber.

\subsection{Impermeability}

Chloride migration coefficient is one of the important indexes to characterize the resistance to chloride ion permeation of cement-based materials. The chloride migration coefficient of C0, CF, CG, and CFG are shown in Figure 4.

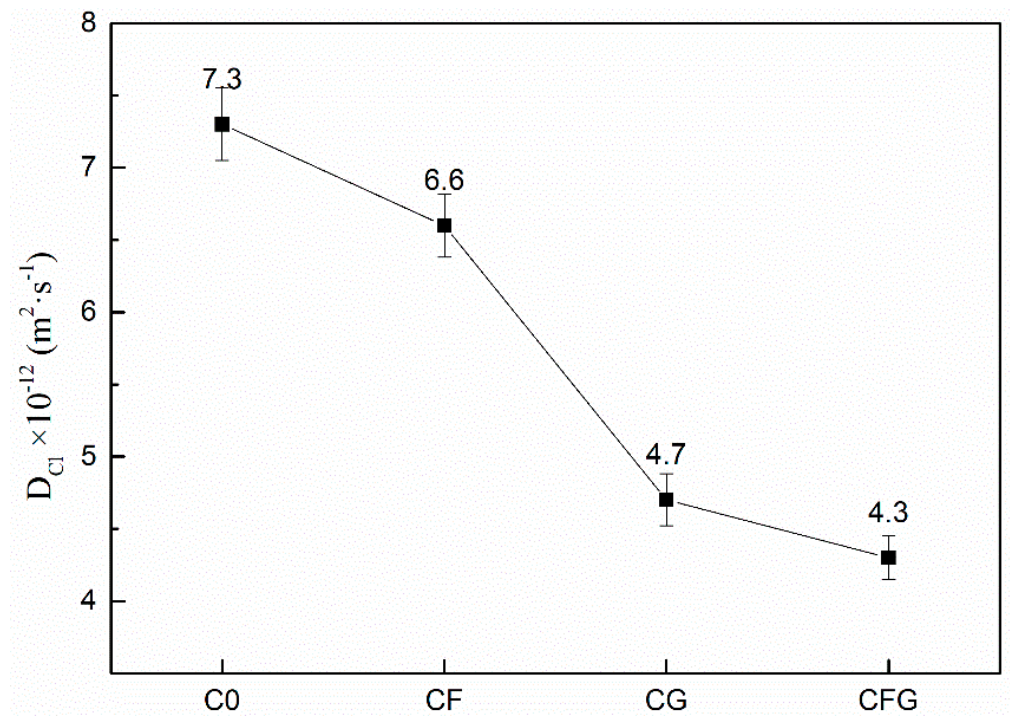

Figure 4. Chloride migration coefficient of samples.

As shown in Figure 4, the chloride migration coefficient of the mixtures was decreased due to the incorporation of PVA fiber and/or GO. Therein, the effect of PVA fiber was minimal, which just led to the chloride migration coefficient decreasing from $7.3 \times 10^{-12} \mathrm{~m}^{2} / \mathrm{s}$ for $\mathrm{C} 0$ to $6.6 \times 10^{-12} \mathrm{~m}^{2} / \mathrm{s}$ for $\mathrm{CF}$, the decrease magnitude was less than $10 \%$. Moreover, the effect of GO was significant; compared with $\mathrm{C} 0$, the chloride migration coefficient of CG was decreased by $35.6 \%$. Furthermore, the coupling effect of PVA fiber and GO resulted in a further decrease in the chloride migration coefficient of CFG, and the decrease magnitude reached $41.1 \%$. 
A previous study [39] revealed that cracks may become paths for chloride ions to penetrate into cement-based materials. PVA fibers mainly act as bridges in the cement matrix, thus restraining the expansion of cracks [36,37], which blocked the paths for chloride ion penetration. As a result, the chloride migration coefficient of CF was slightly decreased. Besides, as stated in previous literature [40-43], chloride ion penetration was closely related to the pore structure of cement-based materials. The chloride migration coefficient showed a linear increase with the pore volume and critical pore diameter. GO in cement-based materials can fill in the micropores and refine the pore structure. Moreover, the nucleation seed effect of it during cement hydration could accelerate the hydration reaction rate, promoting the accumulation of hydration products, thus leading to a denser microstructure with decreased porosity [44]. This explained the reason of the significant decrease in the chloride migration coefficient of CG. As for CFG, on the one hand, PVA fiber inhibited the expansion of the crack, on the other hand, GO refined the microstructure, both actions blocked the path for chloride ion penetration. As a result, the chloride migration coefficient was minimal.

\subsection{Sulfate Corrosion Resistance}

The compressive and flexural strength of $\mathrm{C} 0, \mathrm{CF}, \mathrm{CG}$, and CFG after being immersed in a 5\% $\mathrm{Na}_{2} \mathrm{SO}_{4}$ solution for different times are shown in Figures 5 and 6, respectively.

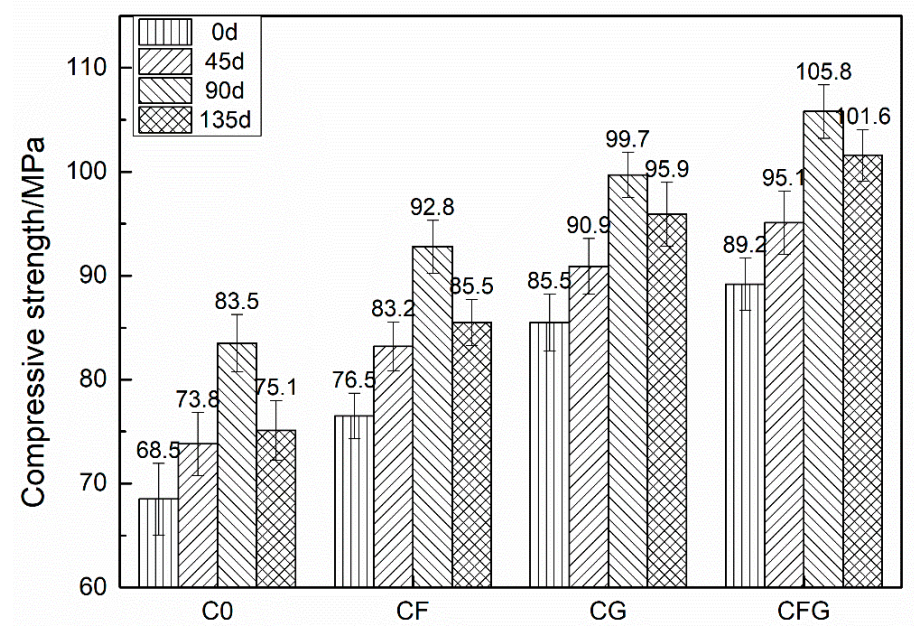

Figure 5. Compressive strength of samples under the sulfate corrosion condition.

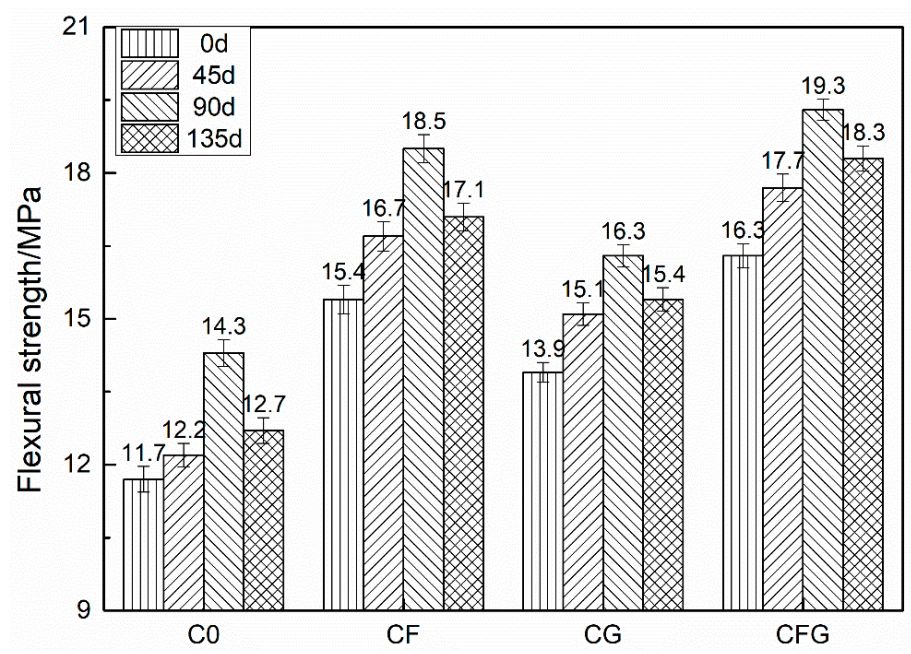

Figure 6. Flexural strength of samples under the sulfate corrosion condition. 
As shown in the figures, the mechanical strength of specimens showed a continuous increase with the soaking time increasing to $90 \mathrm{~d}$. Afterwards, the specimens showed a decreased mechanical strength with the soaking time increasing to $135 \mathrm{~d}$. The mechanical strength of specimens soaked for $135 \mathrm{~d}$ still showed higher mechanical strength compared with that of the specimens before sulfate solution soaking. The values of the compressive strength rates of $\mathrm{C} 0, \mathrm{CF}, \mathrm{CG}$, and CFG were increased from approximately $7 \%$ at $45 \mathrm{~d}$ to $20 \%$ at $90 \mathrm{~d}$ and then decreased to around $11 \%$ at $135 \mathrm{~d}$. The value of the flexural strength increase rate showed a similar trend.

Under sulfate corrosion conditions, the mechanical strength of mortar was mainly affected by two main factors. The positive factor related to the continuous hydration of unhydrated cement in the specimens after $28 \mathrm{~d}$ standard curing, which can produce more hydrates and lead to a more compact mortar matrix with improved mechanical strength. The negative factor was due to the sulfate corrosion process. The $\mathrm{SO}_{4}{ }^{2-}$ ions penetrated into mortar and reacted with the components of the cementitious materials to form intumescent minerals, such as ettringite, resulting in expansion stress and cracks in the mortar with decreased mechanical strength. Both the positive effect due to the continuous hydration and negative effect regarding the sulfate corrosion acted simultaneously. However, at the early stage of sulfate corrosion, the positive factor was dominant, therefore, the mechanical strength of mortar was increased. With the increase of corrosion time, the negative factor became more and more prominent, thus, the mechanical strength begun to decrease.

In addition, as GO could accelerate the hydration reaction rate of cement $[27,28,44]$, the amount of unhydrated cement in the mixture CG or CFG was limited. Consequently, at the early stage, the dominant positive effect due to the continuous hydrations was limited, leading to a minor mechanical strength increase of CG and CFG at $90 \mathrm{~d}$. At the stage of mechanical strength degradation, PVA fibers could counteract the expansion stress and restrain cracks propagation caused by the intumescent minerals. Meanwhile, similar to the case of improved resistance to chloride ion permeability, GO could also improve the resistance to $\mathrm{SO}_{4}{ }^{2-}$ permeability of the mixture, which prevented the formation of intumescent minerals in the mortar. Therefore, due to the coupling action of PVA fiber and GO, the mechanical strength of CFG was maintained at a maximum among the four series of specimens at $135 \mathrm{~d}$.

\subsection{Drying Shrinkage}

Figure 7 shows the shrinkage-time relationship of specimens which developed over a period of $90 \mathrm{~d}$ of air storage. All specimens with the addition of PVA fiber and/or GO were found to exhibit a lower total drying shrinkage than the control sample between 0 to $90 \mathrm{~d}$. With PVA fiber content, the $90 \mathrm{~d}$ shrinkage of CF was decreased by $13.2 \%$ compared with $\mathrm{C} 0$. Moreover, with the incorporation of GO, the $90 \mathrm{~d}$ shrinkage of CG decreased by 17.5\%. Furthermore, when adding PVA fiber coupling with GO, the drying shrinkage of CFG was minimal at all ages and the $90 \mathrm{~d}$ shrinkage was decreased by $22.9 \%$.

The main reason for drying shrinkage was the free water evaporation from the capillary pores through the surface of the cement-based materials, which was exposed to a low RH environment. Capillary forces would hold the free water in the capillary pores. In addition, as a function of pore size diameter, the smaller the pore diameter, the more powerful is the capillary force [45]. In other words, when the pore structure of cement-based materials was more refined, the evaporation of free water is slower, and, thus, the drying shrinkage is smaller. In addition, improving mechanical properties of cement-based materials was also beneficial in the enhancement its ability to resist shrinkage. As discussed before, GO in the cement matrix, on the one hand, filled in the micropores and refined the micropore structure, on the other hand, acted as a nucleation seed to accelerate the hydration reaction rate of cement, thus leading to a denser microstructure, improved mechanical strength [44], and decreased drying shrinkage of mixtures containing GO. Besides, Zhang and Li [46] revealed the possible mechanism for the positive effects of fibers on drying shrinkage: when shrinkage caused tensile stresses in the cement matrix, fibers restrained shrinkage by shear along the fiber-matrix interface. 


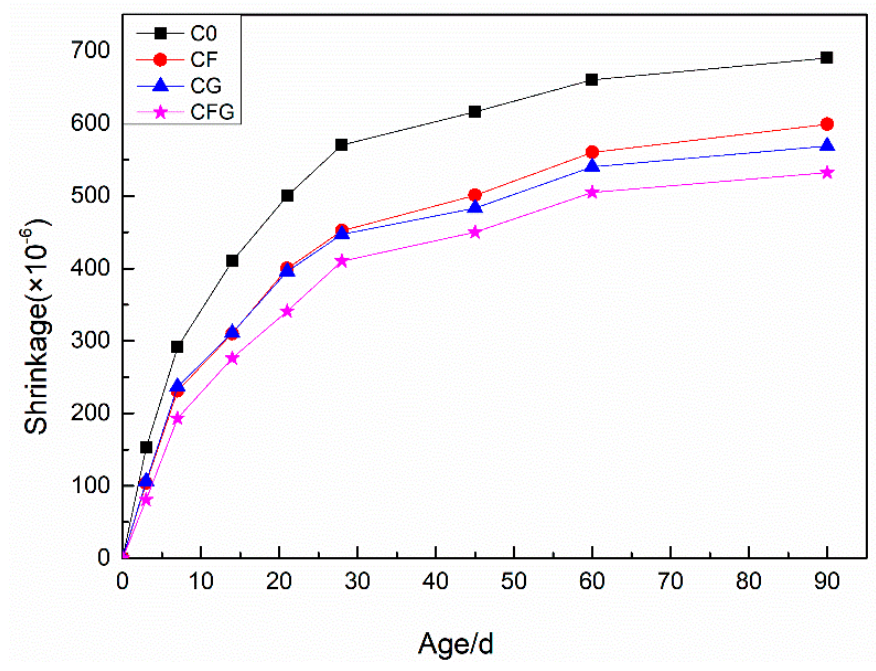

Figure 7. Drying shrinkage rate of specimens at different ages.

\subsection{Pore Structure Analysis}

The pore structure of C0, CF, CG, and CFG at $28 \mathrm{~d}$ was determined by means of Mercury Intrusion Porosimetry (MIP). The porosity and pore size distribution results are summarized in Table 3, and their curves are shown in Figure 8a,b. According to previous research, the pores can be divided into three size domains: $>0.05 \mu \mathrm{m}, 0.05-0.01 \mu \mathrm{m}$, and $<0.01 \mu \mathrm{m}$, which correspond to large capillary pores, medium capillary pores, and gel pores, respectively [47].

As shown in Table 3 and Figure $8 \mathrm{a}$, the cumulative porosity of C0, CF, CG, and CFG were $15.43 \times 10^{-2} \mathrm{~cm}^{3} / \mathrm{g}, 13.23 \times 10^{-2} \mathrm{~cm}^{3} / \mathrm{g}, 3.62 \times 10^{-2} \mathrm{~cm}^{3} / \mathrm{g}$ and $2.67 \times 10^{-2} \mathrm{~cm}^{3} / \mathrm{g}$, respectively. Compared with C0, the cumulative porosity of CF, CG, and CFG was decreased by $14.26 \%, 76.54 \%$, and $82.70 \%$, respectively. Figure $8 \mathrm{~b}$ shows a different pore size distribution for the cement-based materials. The pore structure of $\mathrm{C} 0$ and CF are dominated by large capillary pores, while that of CG and CFG were mainly medium capillary pores. According to Table 3, the proportion of large capillary pores of C0 and CF were $74.40 \%$ and $80.88 \%$, respectively, which decreased to $30.66 \%$ for CG and to $25.84 \%$ for CFG, meanwhile, the proportion of medium capillary pores was increased from $11.48 \%$ for C0 and $10.7 \%$ for CF to $45.58 \%$ for CG and to $59.55 \%$ for CFG. In addition, the total intrusion $\left(\mathrm{cm}^{3} / \mathrm{g}\right)$ of gel pores, usually belonging to $\mathrm{C}-\mathrm{S}-\mathrm{H}$ channels, should increase with the addition of GO, since GO could promote the formation of hydration products. However, the results showed no difference in the gel pore volume between the control specimen and the GO modified specimen. This may be because GO could regulate the crystallization of hydration products [38] and the C-S-H grows within the inter-hydrate spaces (around 8-10 nm), leading to a transition from the growth of "less dense" to "more dense" products [48]. The transition resulted in decreased gel porosity.

The above results indicate that the addition of PVA fiber in cement-based materials only showed a slight effect on pore structure, but GO incorporation can significantly decrease the porosity and refine the pore size. It also proved the statement discussed in the previous section that GO in cement-based materials could not only fill in the micropores but also act as crystallization centers for hydrated products to accelerate the hydration reaction rate, leading to a refined microstructure. 


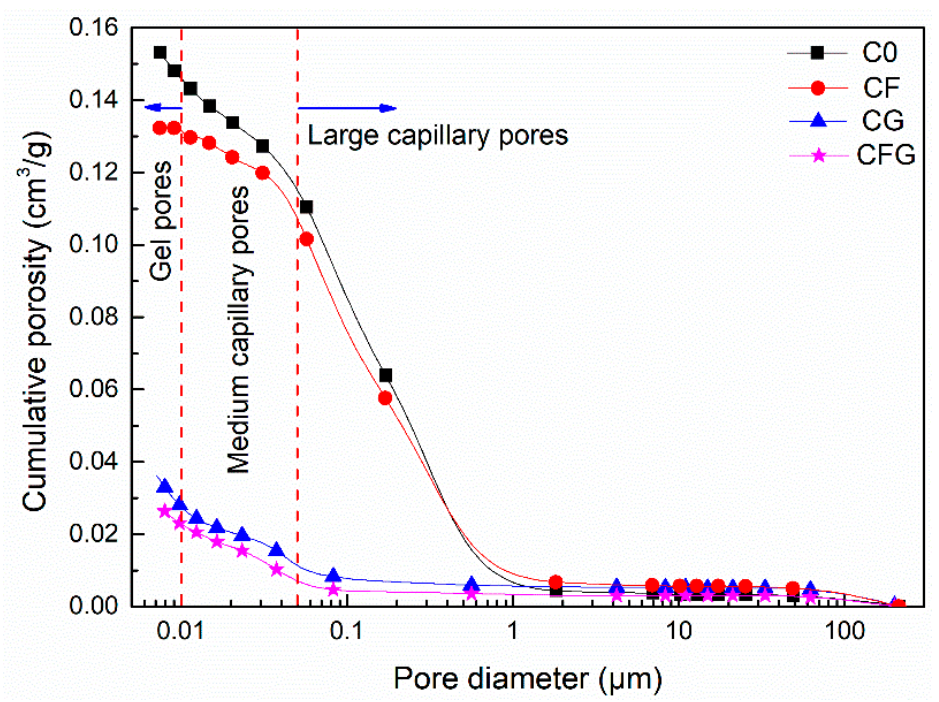

(a)

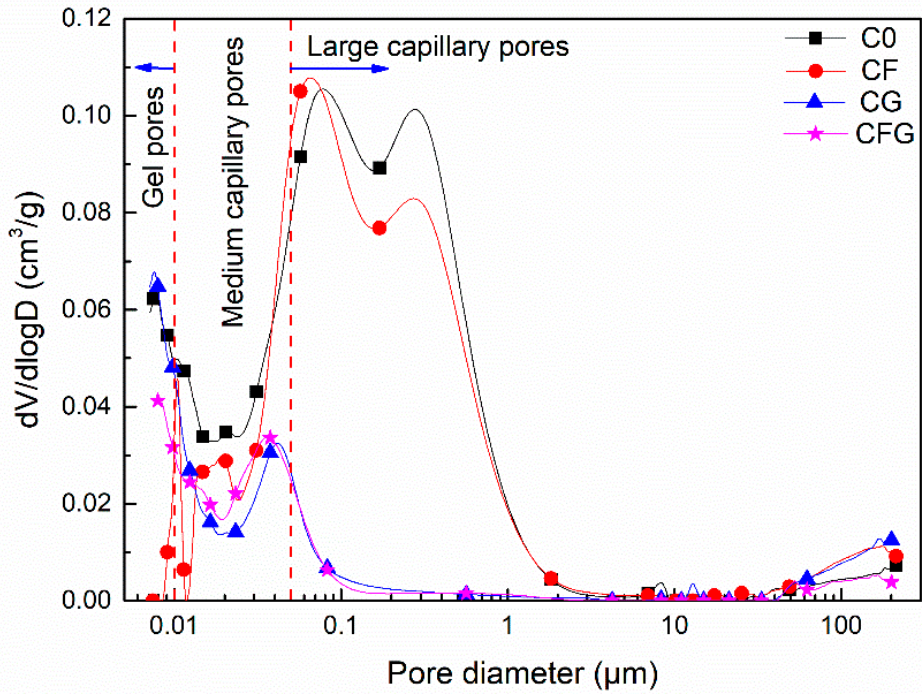

(b)

Figure 8. (a) Cumulative porosity; and (b) Log-differential volume curve plots.

Table 3. Porosity and pore size distribution of samples.

\begin{tabular}{|c|c|c|c|c|c|c|c|}
\hline \multirow{3}{*}{ Samples } & \multirow{3}{*}{$\begin{array}{c}\text { Porosity } \\
10^{-2} \mathrm{~cm}^{3} / \mathrm{g}\end{array}$} & \multicolumn{6}{|c|}{ Pore Size } \\
\hline & & \multicolumn{2}{|c|}{$<0.01 \mu \mathrm{m}$} & \multicolumn{2}{|c|}{$0.01 \sim 0.05 \mu \mathrm{m}$} & \multicolumn{2}{|c|}{$>0.05 \mu \mathrm{m}$} \\
\hline & & $10^{-2} \mathrm{~cm}^{3} / \mathrm{g}$ & $\%$ & $10^{-2} \mathrm{~cm}^{3} / \mathrm{g}$ & $\%$ & $10^{-2} \mathrm{~cm}^{3} / \mathrm{g}$ & $\%$ \\
\hline $\mathrm{CO}$ & 15.43 & 0.85 & 5.51 & 3.10 & 20.09 & 11.48 & 74.40 \\
\hline $\mathrm{CF}$ & 13.23 & 0.08 & 0.60 & 2.45 & 18.52 & 10.7 & 80.88 \\
\hline CG & 3.62 & 0.86 & 23.76 & 1.65 & 45.58 & 1.11 & 30.66 \\
\hline CFG & 2.67 & 0.39 & 14.61 & 1.59 & 59.55 & 0.69 & 25.84 \\
\hline
\end{tabular}

\subsection{SEM Observations}

Microstructure morphology of hardened specimens at $28 \mathrm{~d}$ was evaluated by means of SEM. Two images were captured of each specimen at different positions. The results are shown in Figure 9. 

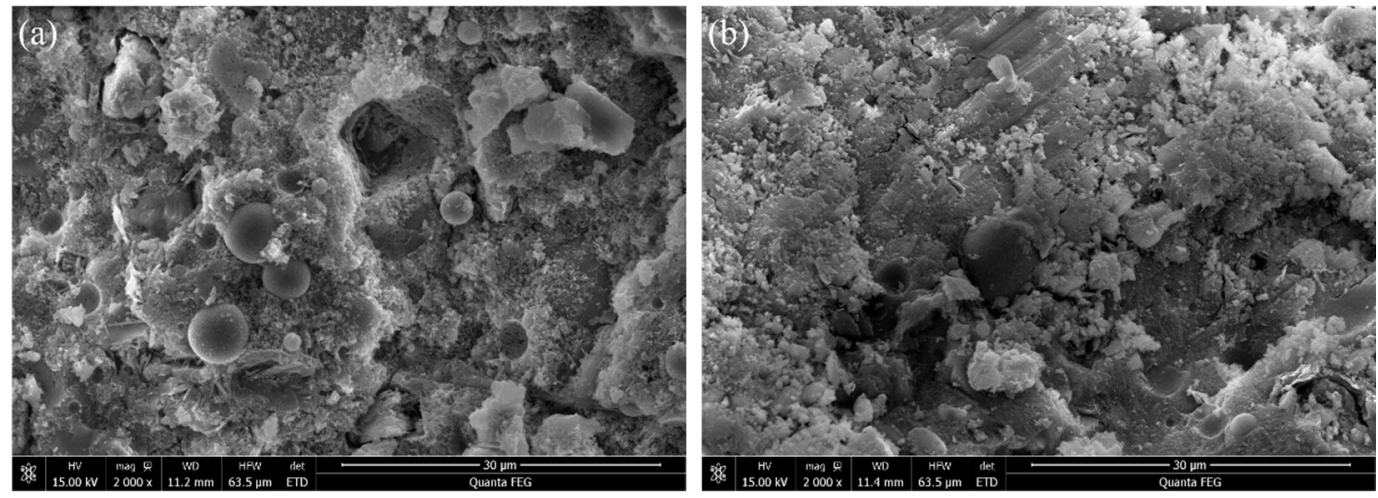

(C0)
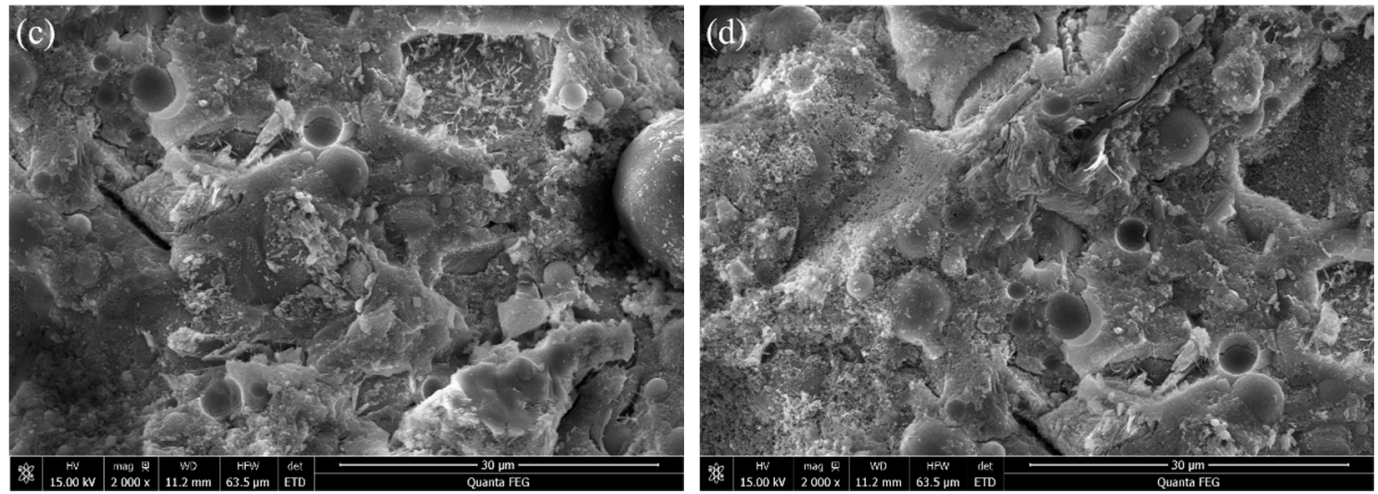

(CG)
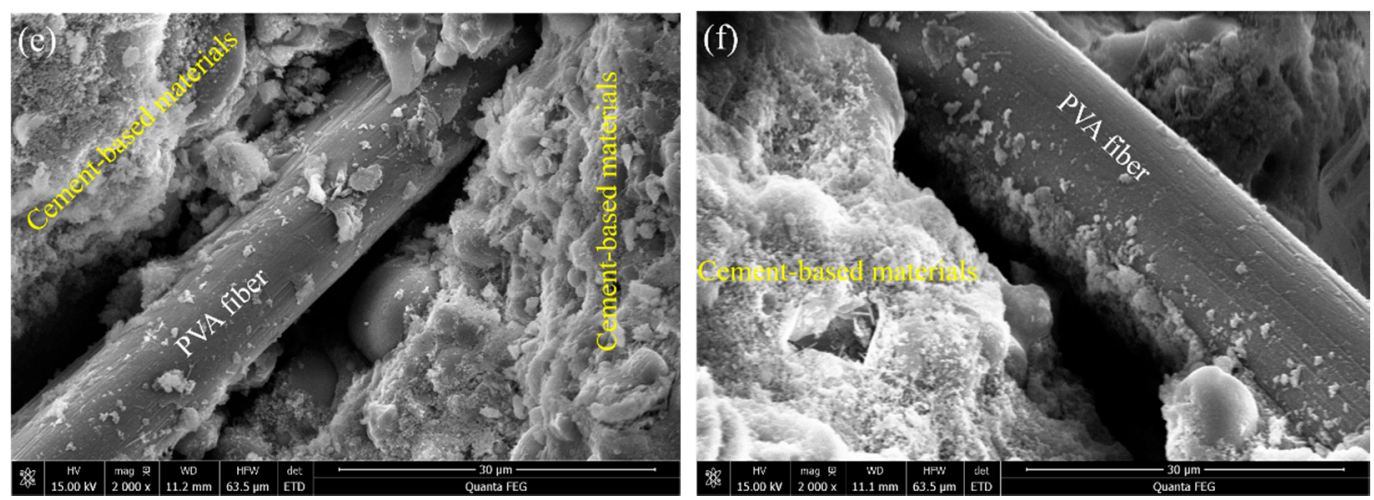

(CF)
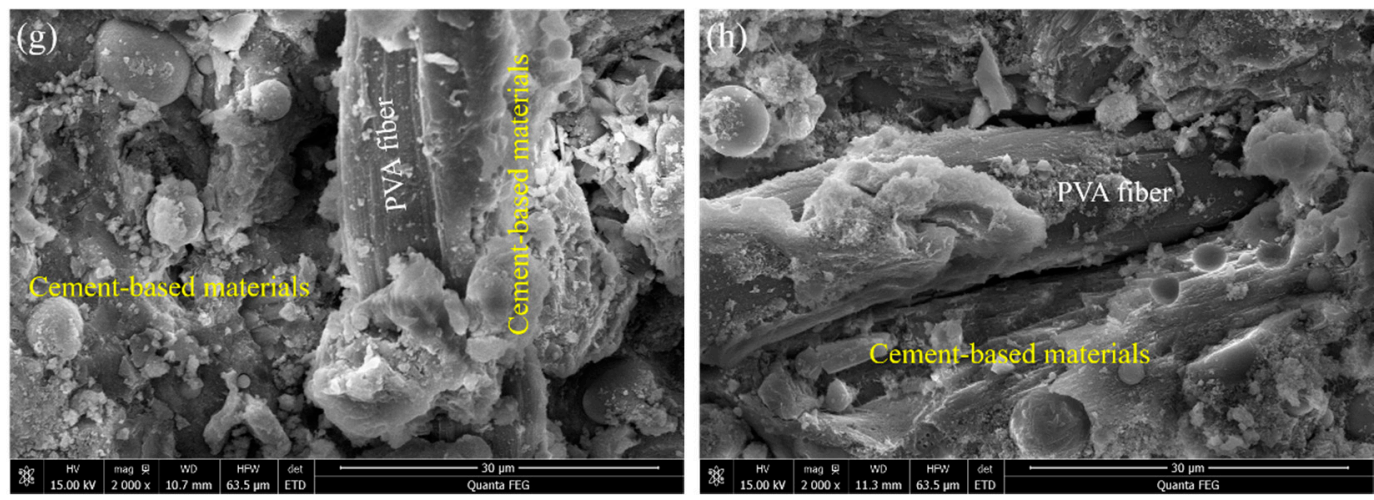

(CFG)

Figure 9. Microstructure morphology of hardened specimens at $28 \mathrm{~d}$ age at 2000 magnification; $(\mathbf{a}, \mathbf{b})$ control sample; (c,d) GO modified sample; (e,f) PVA fiber modified sample; (g,h) GO coupling with PVA fiber modified sample. 
As shown in Figure 9a,b, there was loose-pellet-like C-S-H gel crystal nucleuses and unhydrated FA particles in the microstructure of $\mathrm{C} 0$. Comparatively, Figure 9c,d show a compact and uniform microstructure of CG. The observation indicated that with the addition of GO, the microstructure of the cement matrix was refined, which can be due to GO being involved in the cement hydration, accelerating the nucleation, growth, and phase separation process of hydrated products, thereby promoting the hydration rate and improving the crystal order [38]. Moreover, differences between Figure $9 \mathrm{e}, \mathrm{f}$ and Figure $9 \mathrm{~g}, \mathrm{~h}$ can be observed by comparing carefully. Figure $9 \mathrm{~g}$, h show not only a compact microstructure of cement matrix but also abundant hydration products adhering to the PVA fiber surface. These micrographs reveal that GO in PVA fiber modified cement-based materials could promote the formation of hydration products to densify the microstructure and improve the adhesion of hydration products on PVA fiber, which are beneficial to the combination between PVA fiber and the cement matrix.

\section{Conclusions}

In this study, the influence on the properties of mortar modified by PVA fibers and GO, including mechanical properties, durability and pore structure, were investigated. Based on the results obtained, the following conclusions can be drawn.

PVA fiber in cement-based materials can effectively improve their toughness and ability of resist crack, thus resulting in an evident improvement of mechanical strength. The inclusion of GO in cement systems was beneficial for pore modification, which in turn enhanced the mechanical and durability properties of the cement-based materials. Moreover, the mechanical and durability properties of cement-based materials can be further improved by the coupling modification of PVA fiber and GO. With the addition of both PVA fiber and GO, the compressive and flexural strength of mortar at $28 \mathrm{~d}$ was increased by $30.2 \%$ and $39.3 \%$, respectively; meanwhile, the durability properties, including chloride ion impermeability, sulfate corrosion resistance, and drying shrinkage property, were clearly improved.

It can be found from the MIP analysis that the addition of GO in cement-based materials could lead to a decreased cumulative porosity, the value of the decrease rate was about $80 \%$, compared with $\mathrm{C} 0$. Furthermore, the addition of GO can refine the pore structure, which means the proportion of large capillary pores reduced from around $80 \%$ to $30 \%$ and the proportion of medium capillary pores and gel pores, respectively, increased from approximately $20 \%$ and $5 \%$ to $50 \%$ and $20 \%$ with the incorporation of GO.

The SEM images showed that the addition of GO in cement-based materials made the microstructure more compact and uniform, meanwhile, it improved the adhesion of hydration products on the PVA fiber, which was beneficial to the combination between PVA fiber and the cement matrix. The mechanical strength of the PVA fiber coupling with the GO modified specimen was higher than that of the PVA fiber without the GO modified specimen.

Since the cement-based materials modified by GO coupling with PVA fiber exhibited excellent mechanical strength and durability, it can be widely used in buildings and structures, such as roads, bridges, dams, etc.

Author Contributions: W.J. did the experiments, data analysis and wrote part of this paper. X.L. provided the original ideas, wrote part of this paper and participated in the revision work. Y.L. did the data analysis and participated in the revision work. M.Z. and Z.L. guided the tests. Z.R. did the experiments. Z.Y. provided the original ideas.

Funding: This research was funded by National Natural Science Foundation of China $(51572207,51378408)$ and State Key Laboratory of Silicate Materials for Architectures (Wuhan University of Technology) (SYSJJ2016-03).

Acknowledgments: The authors are grateful for the financial support from National Natural Science Foundation of China $(51572207,51378408)$ and State Key Laboratory of Silicate Materials for Architectures (Wuhan University of Technology) (SYSJJ2016-03).

Conflicts of Interest: We declare that there is no conflict of interest in this work. 


\section{References}

1. Mokhtar, M.M.; Abo-El-Enein, S.A.; Hassaan, M.Y.; Morsy, M.S.; Khalil, M.H. Mechanical performance, pore structure and micro-structural characteristics of graphene oxide nano platelets reinforced cement. Constr. Build. Mater. 2017, 138, 333-339. [CrossRef]

2. Li, X.; Liu, Y.M.; Li, W.G.; Li, C.Y.; Sanjayan, J.G.; Duan, W.H.; Li, Z. Effects of graphene oxide agglomerates on workability, hydration, microstructure and compressive strength of cement paste. Constr. Build. Mater. 2017, 145, 402-410. [CrossRef]

3. Ali, M.; Li, X.; Chouw, N. Experimental investigations on bond strength between coconut fibre and concrete. Mater. Des. 2013, 44, 596-605. [CrossRef]

4. Zhang, M.H.; Li, H. Pore structure and chloride permeability of concrete containing nano-particles for pavement. Constr. Build. Mater. 2011, 25, 608-616. [CrossRef]

5. Bentur, A.; Mindess, S. Fibre reinforced cementitious composites. Mater. Build. Str. 2006, 6, 726-732.

6. Chan, Y.W.; Chu, S.H. Effect of silica fume on steel fiber bond characteristics in reactive powder concrete. Cement Concr. Res. 2004, 34, 1167-1172. [CrossRef]

7. Silva, F.D.A.; Mobasher, B.; Soranakom, C.; Filho, R.D.T. Effect of fiber shape and morphology on interfacial bond and cracking behaviors of sisal fiber cement based composites. Cement Concr. Compos. 2011, 33, 814-823. [CrossRef]

8. Mobasher, B.; Li, C.Y. Effect of interfacial properties on the crack propagation in cementitious composites. Adv. Cement Based Mater. 1996, 4, 93-105. [CrossRef]

9. Naaman, A.E.; Najm, H. Bond-slip mechanisms of steel fibers in concrete. Aci Mater. J. 1991, 88, $135-145$.

10. Shannag, M.J.; Brincker, R.; Hansen, W. Pullout behavior of steel fibers from cement-based composites. Cement Concr. Res. 1997, 27, 925-936. [CrossRef]

11. Singh, S.; Shukla, A.; Brown, R. Pullout behavior of polypropylene fibers from cementitious matrix. Cement Concr. Res. 2004, 34, 1919-1925. [CrossRef]

12. Man, D.L. Study on affection of the PVA fiber on recycled coarse aggregate to porous concrete performance. Appl. Mech. Mater. 2013, 438-439, 304-308. [CrossRef]

13. Nguyen, D.D.; Devlin, L.P.; Koshy, P.; Sorrell, C.C. Effects of chemical nature of polyvinyl alcohol on early hydration of portland cement. J. Therm. Anal. Calorim. 2015, 123, 1-12. [CrossRef]

14. Thong, C.C.; Teo, D.; Ng, C.K. Application of polyvinyl alcohol (PVA) in cement-based composite materials: A review of its engineering properties and microstructure behavior. Constr. Build. Mater. 2016, 107, 172-180. [CrossRef]

15. Garcia, S.; Naaman, A.E.; Pera, J. Experimental investigation on the potential use of poly(vinyl alcohol) short fibers in fiber-reinforced cement-based composites. Smart Mater. Str. 1997, 30, 43-52. [CrossRef]

16. Bonapasta, A.A.; Buda, F.; Colombet, P. Cross-linking of poly(vinyl alcohol) chains by al ions in macro-defect-free cements: A theoretical study. Chem. Mater. 2000, 12, 738-743. [CrossRef]

17. Schmidt, M.; Amrhein, K.; Braun, T.; Glotzbach, C.; Kamaruddin, S.; Tanzer, R. Nanotechnological improvement of structural materials-Impact on material performance and structural design. Cement Concr. Compos. 2013, 36, 3-7. [CrossRef]

18. Amin, M.; El-Hassan, K.A. Effect of using different types of nano materials on mechanical properties of high strength concrete. Constr. Build. Mater. 2015, 80, 116-124. [CrossRef]

19. Nirmala, J.; Dhanalakshmi, G. Influence of nano materials in the distressed retaining structure for crack filling. Constr. Build. Mater. 2015, 88, 225-231. [CrossRef]

20. Li, H.; Xiao, H.G.; Yuan, J.; Ou, J. Microstructure of cement mortar with nano-particles. Compos. Part B Eng. 2004, 35, 185-189. [CrossRef]

21. Stefanidou, M.; Papayianni, I. Influence of nano-SiO 2 on the Portland cement pastes. Compos. Part B 2012, 43, 2706-2710. [CrossRef]

22. Ji, T. Preliminary study on the water permeability and microstructure of concrete incorporating nano-SiO 2 . Cement Concr. Res. 2005, 35, 1943-1947. [CrossRef]

23. Kong, D.; Du, X.; Wei, S.; Zhang, H.; Yang, Y.; Shah, S.P. Influence of nano-silica agglomeration on microstructure and properties of the hardened cement-based materials. Constr. Build. Mater. 2012, 37, 707-715. [CrossRef] 
24. Kang, S.T.; Seo, J.Y.; Park, S.H. The characteristics of CNT/Cement composites with acid-treated MWCNTs. Adv. Mater. Sci. Eng. 2015, 2015, 1-9. [CrossRef]

25. Li, X.; Wei, W.; Qin, H.; Hu, Y.H. Co-effects of graphene oxide sheets and single wall carbon nanotubes on mechanical properties of cement. J. Phys. Chem. Solids 2015, 85, 39-43. [CrossRef]

26. Chen, J.; Yao, B.; Li, C.; Shi, G. An improved Hummers method for eco-friendly synthesis of graphene oxide. Carbon 2013, 64, 225-229. [CrossRef]

27. Peng, H.X.; Guo, X.L.; Liu, Z.L.; Yang, R.; Wei, J.D. Influence of graphene oxide on hydration and mechanical properties of fly ash-cement. Bull. Chin. Ceram. Soc. 2016, 73, 113-124.

28. Li, W.G.; Li, X.Y.; Chen, S.J.; Liu, Y.M.; Duan, W.H.; Shah, S.P. Effects of graphene oxide on early-age hydration and electrical resistivity of Portland cement paste. Constr. Build. Mater. 2017, 136, 506-514. [CrossRef]

29. Pan, Z.; He, L.; Qiu, L.; Korayem, A.H.; Li, G.; Zhu, W.J.; Collins, F.; Li, D.; Duan, H.W.; Wang, M.C.; et al. Mechanical properties and microstructure of a graphene oxide-cement composite. Cement Concr. Compos. 2015, 58, 140-147. [CrossRef]

30. Lv, S.; Ma, Y.; Qiu, C.; Sun, T.; Liu, J.; Zhou, Q. Effect of graphene oxide nanosheets of microstructure and mechanical properties of cement composites. Constr. Build. Mater. 2013, 49, 121-127. [CrossRef]

31. Lin, C.; Wei, W.; Hu, Y.H. Catalytic behavior of graphene oxide for cement hydration process. J. Phys. Chem. Solids 2016, 89, 128-133. [CrossRef]

32. Mohammed, A.; Sanjayan, J.G.; Duan, W.H.; Nazari, A. Graphene oxide impact on hardened cement expressed in enhanced freeze-thaw resistance. J. Mater. Civ. Eng. 2016, 8. [CrossRef]

33. Li, X.G.; Liu, Z.L.; Lv, Y.; Zhou, Y. Influence of graphene oxide on hydration characteristics of tricalcium silicate. Adv. Cement Res. 2018. [CrossRef]

34. National Standard of the People's Republic of China. Standard for Test Method of Mechanical Properties on Ordinary Concrete (GB/T 50081-2002); Ministry of Construction of the People's Republic of China: Beijing, China, 2002.

35. National Standard of the People's Republic of China. Standard for Test Methods of Long-term Performance and Durability of Ordinary Concrete (GB/T 50082-2009); Ministry of Construction of the People's Republic of China: Beijing, China, 2009.

36. Noushini, A.; Samali, B.; Vessalas, K. Flexural Toughness and Ductility Characteristics of Polyvinyl-Alcohol Fibre Reinforced Concrete (PVA-FRC). In Proceedings of the 8th International Conference on Fracture Mechanics of Concrete and Concrete Structures (FraMCo-8), Toledo, Spain, 10-14 March 2013; pp. 1110-1121.

37. Atahan, H.N.; Tuncel, E.Y.; Pekmezci, B.Y. Behavior of PVA Fiber-Reinforced Cementitious Composites under Static and Impact Flexural Effects. J. Mater. Civ. Eng. 2013, 25, 1438-1445. [CrossRef]

38. Wang, Q.; Wang, J.; Lu, C.-X.; Liu, B.-W.; Zhang, K.; Li, C.-Z. Influence of graphene oxide additions on the microstructure and mechanical strength of cement. New Carbon Mater. 2015, 30, 349-356. [CrossRef]

39. Kobayashi, K.; Kojima, Y. Effect of fine crack width and water cement ratio of SHCC on chloride ingress and rebar corrosion. Cement Concr. Compos. 2017, 80, 235-244. [CrossRef]

40. Yang, C.C. On the relationship between pore structure and chloride diffusivity from accelerated chloride migration test in cement-based materials. Cement Concr. Res. 2006, 36, 1304-1311. [CrossRef]

41. Gao, L.; Sun, G.; Zhang, Y.; Sun, W. Relationship between porosity and chloride diffusivity in cement-based composite materials. J. Chongqing Univ. 2012, 35, 53-61.

42. Halamickova, P.; Detwiler, R.J.; Bentz, D.P.; Garboczi, E.J. Water permeability and chloride ion diffusion in portland cement mortars: Relationship to sand content and critical pore diameter. Cement Concr. Res. 1995, 25, 790-802. [CrossRef]

43. Zhang, B.; Tan, H.; Shen, W.; Xu, G.; Ma, B.; Ji, X. Nano-silica and silica fume modified cement mortar used as surface protection material to enhance the impermeability. Cement Concr. Compos. 2018, 92, 7-17. [CrossRef]

44. Yang, H.; Monasterio, M.; Cui, H.; Han, N. Experimental study of the effects of graphene oxide on microstructure and properties of cement paste composite. Compos. Part A Appl. Sci. Manuf. 2017, 102, 263-272. [CrossRef]

45. Aitcin, P.C.; Neville, A.M.; Acker, P. Integrated view of shrinkage deformation. Concr. Int. 1997, 19, 35-41.

46. Zhang, P.; Li, Q.F. Effect of polypropylene fiber on durability of concrete composite containing fly ash and silica fume. Compos. Part B 2013, 45, 1587-1594. [CrossRef] 
47. Shaikh, F.U.A.; Supit, S.W.M. Chloride induced corrosion durability of high volume fly ash concretes containing nano particles. Constr. Build. Mater. 2015, 99, 208-225. [CrossRef]

48. Muller, A.C.A.; Scrivener, K.L.; Gajewicz, A.M.; McDonald, P.J. Densification of C-S-H measured by H NMR relaxometry. J. Phys. Chem. C 2013, 117, 403-412. [CrossRef] 